\title{
Event-related potentials to odor stimuli
}

\author{
TYLER S. LORIG, AMY C. SAPP, JAMIE CAMPBELL \\ Washington and Lee University, Lexington, Virginia \\ and \\ WILLIAM S. CAIN \\ John B. Pierce Laboratory and Yale University, New Haven, Connecticut
}

\begin{abstract}
Fifteen subjects participated in a study designed to record event-related potentials (ERPs) to various concentrations of the odorant $n$-butyl alcohol. Subjects pressed a response key upon detection of the odor, while ERPs were recorded from nine electrode positions. Results indicated a concentration-dependent increase in amplitude of a P300-like component 320-520 msec following the stimulus onset. Correlations between subjects' sensory thresholds and activity at frontal electrode sites were also found. The absence of early ERP components is discussed.
\end{abstract}

Interest in olfactory event-related potentials (ERPs) began more than two decades ago with the pioneering work of Allison and Goff (1967). These investigators realized the potential clinical usefulness of such measurements, even in the lower senses. Since that time, clinical interest in smell has grown considerably and so has the need for objective ways to demonstrate the existence of olfaction in patients suffering certain ailments. In the United States, approximately 10 clinics devote their efforts to the diagnosis and treatment of olfactory and taste disorders. Approximately $10 \%$ of patients who visit such clinics, and an undetermined number who visit otolaryngologists and neurologists, claim to have lost smell through head trauma. Commonly, the patients are engaged in personal injury litigation, and in such instances, the existence of an objective measure to validate the loss or confirm the presence of smell would prove invaluable.

Clinical research has revealed progressive olfactory dysfunction in various diseases, including Alzheimer's, Parkinsonism, and Korsakoff's Syndrome, that may include dementia among their symptoms (Doty, Deems, \& Stellar, 1988; Jones, Moskowitz, \& Butters, 1975; Koss, 1986). In Alzheimer's disease, for instance, the neurofibrillary tangles and plaques characteristic of the disease appear first in the olfactory tract. A sensitive objective index of olfactory changes could serve for both research and clinical evaluation. Although some patients in the early stages of the disorder can cooperate in psychophysical testing, progressive cognitive loss eventually takes its toll on the validity of subsequent findings.

\footnotetext{
The authors wish to thank the R.E. Lee Research Fund of Washington and Lee University for the support of A.C.S. and J.C. The authors are also indebted to $\mathrm{L}$. Farwell for his suggestions concerning the response measure used in this study and to E. Cometto-Muniz for his assistance with the chromatography. Correspondence should be addressed to T. S. Lorig, Department of Psychology, Washington and Lee University, Lexington, VA 24450.
}

Allison and Goff (1967) reported an objective technique for testing olfactory function. When these investigators recorded potentials in response to the presentation of pulsate olfactory stimuli, they found it difficult to demonstrate that the potentials came strictly from olfactory-induced activity rather than from somesthetically induced activity mediated by stimulation of the trigeminal nerve. More recent research has convincingly met criteria for an olfactory origin; in this newer work, the use of pulsate stimulation techniques has been avoided. For instance, Plattig and Kobal (1979) argued effectively against such an odor delivery procedure and suggested that odorized air should be delivered in a continuous stream. Kobal and colleagues (Kobal \& Hummel, 1988; Kobal, Hummel, \& van Toller, 1987) reported successful collection of odor ERPs with the use of this continuous flow technique. Tubes delivered the odorized air in the nasal cavity to a point near the mucosa; with the tubes occupying the nasal cavity, the subjects breathed through the mouth. The odors were then presented to the subjects independently of breathing. Kobal and Hummel (1988) reported that odors presented in this way produced a large N100/P200 component at the vertex; the component varied with the odor administered. Although it produces reliable odor ERPs, this technique requires that subjects use an atypical style of breathing, and such breathing may be problematic in some subjects and may lead to a difficulty in reconciling data from the psychophysical and psychophysiological domains.

Freeman and Schneider (1982) have found that the olfactory bulb of rabbits produces a pattern of identifiable electrical activity synchronized with nasal respiration. They suggest that an "odor template" is placed on the bulb prior to the inhalation of an odor. If this is also the case for humans, the ERP obtained with natural inhalations may differ from that obtained using a technique in which odors are presented out of phase with respiration. Furthermore, if ERPs are to serve as an objective index of olfactory activity in patients who cannot or will not cooperate in the use of the special breathing technique, 
it would seem desirable to collect ERPs only during natural inhalations.

An early effort to collect odor ERPs under somewhat more natural conditions involved synchronizing EEG data collection to the inhalation cycle while odors were presented continuously (Lorig, Herman, \& Schwartz, 1988). Although the amplitude of several of the resulting ERP components correlated with self-report measures, subsequent efforts to replicate the waveform were unsuccessful. Other studies that followed this initial effort were also unsuccessful in producing reliable odor ERPs, although they helped identify variables important to their collection. Several of the parametric variables that were determined to be of importance in these pilot studies included the rate and consistency of nasal inhalation, the rapidity of odor onset and offset, and implementation of techniques to reduce eye movements, especially blinking. The study described here employed these parameters in obtaining reliable odor ERPs.

\section{METHOD}

\section{Subjects}

Fifteen individuals (6 males, 9 females) volunteered to participate in the study. The group was relatively diverse, representing high school and college students, and college faculty and staff. The subjects ranged in age from 17 to 54 years $(M=29.1)$. All were right-handed and were screened for health problems before participating in the study.

\section{Odor Testing}

After giving their informed consent, the subjects were tested for olfactory functioning on the Connecticut Chemosensory Clinical Research Center odor test (CCCRC) (Cain, 1989). This test consists of a threshold component with an 11-step dilution series of 1-butyl alcohol and an odor identification component with seven common odors (baby powder, peanut butter, soap, etc.). The highest concentration of 1-butyl alcohol was $4 \%$ in water, and the series steps were in successive thirds. Threshold testing followed a two-alternative forced choice procedure that progressed from weaker to stronger concentrations. A criterion of five correct choices in a row was used to establish the thresholds (see Cain \& Rabin, 1989).

\section{ERP Data Collection}

ERP data were collected from nine electrode locations (F7, T3, T5, $\mathrm{Fz}, \mathrm{Cz}, \mathrm{Pz}, \mathrm{F8}, \mathrm{T} 4$, and T6), where 10-mm cup-type electrodes were affixed with Ellefix electrode paste. Linked mastoids served as reference to the active sites and electrode impedance was always below $10 \mathrm{k} \Omega$ and typically below $5 \mathrm{k} \Omega$. Grass Model 8A5 amplifiers set to a bandpass of $0.3-35 \mathrm{~Hz}$ amplified the signal, which was stored on line at a rate of 166 samples/sec for each channel. Eye movements were recorded from electrodes over the right eye. Buttonpresses used to signal the detection of the odor and nasal respirations (thermocouple) were recorded simultaneously and stored with the ERP data. The stimulating and recording equipment was located in a room immediately adjacent to the subject.

\section{Odor Administration}

The odors were delivered through an olfactometer to a small, lightweight oxygen mask. The mask was modified by removing portions of both sides to allow good ventilation and exhaust of odors. Teflon tubing connected the mask to the olfactometer. Manually operated valves in the olfactometer allowed selection of one of four odor-containing vessels through which charcoal-filtered room air was passed. A three-way, quiet solenoid valve (ASCO 8320B174Q) could be triggered by the computer to cause odorized air to be added to the constant air flow directed to the mask. The flow rate for all experimental conditions was $2,000 \mathrm{ml} / \mathrm{min}$ administered through a 2-mm (ID) orifice. Because this flow rate was insufficient to produce any nasal drying, the airstream was not humidified. The temperature of the air flow was $22^{\circ} \mathrm{C}$. Three aqueous con-

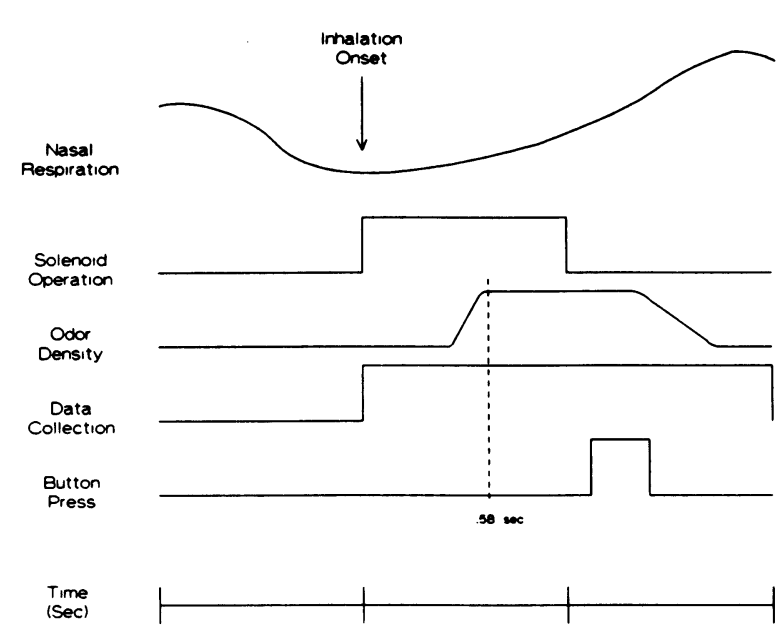

Figure 1. Timing parameters for stimulus delivery and data collection.

centrations of 1-butyl alcohol were used $(4 \%, 1.33 \%$, and $0.444 \%)$. Two milliliters of each concentration and a fourth stimulus of distilled water were placed in separate channels inside the olfactometer. The three odor conditions produced vapor-phase concentrations of $1,647,550$, and $183 \mathrm{ppm}$, respectively, at the subject's nose. A Hewlett-Packard 5890 gas chromatograph equipped with a gas sampling valve and photoionization detector was used to establish vapor phase concentration.

\section{Procedure}

After the electrodes were affixed to the scalp, the subjects were exposed to all odor concentrations for $2 \mathrm{sec}$ each in order to familiarize them with the stimuli. The odors were then readministered, and the subjects were asked to rate the intensity and pleasantness of each concentration by using a line bisection scale presented on a computer monitor $1 \mathrm{~m}$ in front of them. The subjects moved a joystick and buttonpressed to indicate their ratings. After such ratings, the ERP portion of the experiment began. In this phase, the subjects were instructed to press the joystick button with their right hands whenever they smelled an odor. Instructions presented a few seconds prior to each trial told the subjects that a trial was upcoming and that an odor might be presented. They were further instructed to close their eyes and press only if they smelled an odor. A tone signaled the availability of instructions. The eyes closed condition was used since pilot studies indicated a tendency for subjects to blink simultaneously with buttonpresses. Although eye-movement correction algorithms reduced the problem, the occurrence of so many blinks at precisely the same time point in the ERP produced artifacts in the earlier averages.

Each of the four odor concentrations was presented 18 times in random order. The mean intertrial interval was $20 \mathrm{sec}$, during which odor was evacuated from the mask by the constant air flow. The odor duration during the ERP phase was $1 \mathrm{sec}$. The timing for the delivery of the stimuli and the synchronization with the onset of each trial was determined by the subjects' respiratory patterns. Previous pilot data indicated that inspirations associated with attempts to smell an odor tend to be much more consistent than resting inspiratory patterns. Therefore, the subjects were told when a trial was upcoming, and computer averaging and odor delivery were initiated on a pattern of exhalation followed by inhalation. The timing parameters of odor availability and data collection are presented in Figure 1.

As is indicated in the figure, the stimuli and ERP averages began with the onset of nasal inhalations. Parametric studies of the operation of the olfactometer using detection of smoke by an optical densitometer indicated that it took $0.42 \mathrm{sec}$ for gas to reach the subject and that the peak concentration was available after $0.58 \mathrm{sec}$. Ambient white noise ( $56 \mathrm{~dB} \mathrm{~A}$ ) was provided throughout the ERP phase of the experiment. Testing was conducted in one 1.5-h session. After data collection, the subjects were debriefed and dismissed. 


\section{RESULTS AND DISCUSSION}

The results of the CCCRC testing indicated that all the subjects were normosmic with a mean odor threshold at Step 6.33 for the left nostril and Step 6.93 for the right. Self-reports of odor intensity increased monotonically with increases in concentration for the four test levels (see Figure 2). Rated pleasantness was highest for the $0.44 \%$ concentration and decreased at higher concentrations (see Figure 2).

Single trials of ERP data were corrected for eye movements (Elbert, Lutzenberger, Rockstroh, \& Birbaumer, 1985) and spatially filtered (Hjorth, 1980) to increase the precision of spatial estimates of brain activity. The 18 trials in each condition were averaged for each subject, though trials with excessive amplitude artifacts were excluded ( $20 \%$ of the ERP in excess of $40 \mu \mathrm{V}$ ). After the averages had been obtained, the data for all the subjects and the intensity levels were submitted to principal components analysis with varimax rotation (BMDP 4M). The results of this analysis indicated that only two ERP components occurred prior to the subjects' detection of the odors. The primary component of the PCA was greatest at the beginning of the ERP; it decreased slowly with time, revealing no identifiable peak. The second component of the PCA indicated a consistent positive peak in the wave, occurring between 0.9 and $1.1 \mathrm{sec}$ poststimulus $(0.32-0.52 \mathrm{sec}$ after the odor reached full strength). Peaks and latencies were obtained for this temporal window and correlated with subjects' scores on threshold testing. Because the
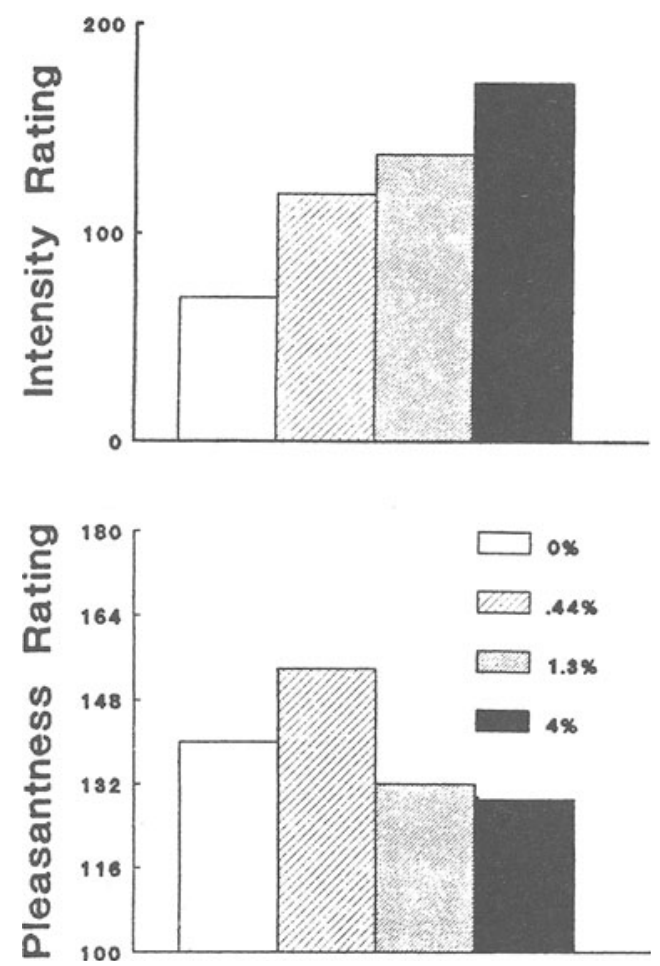

Figure 2. Self-report ratings of perceived intensity (left panel) and pleasantness (right panel) for the four odor concentrations.
Side of Stimulation
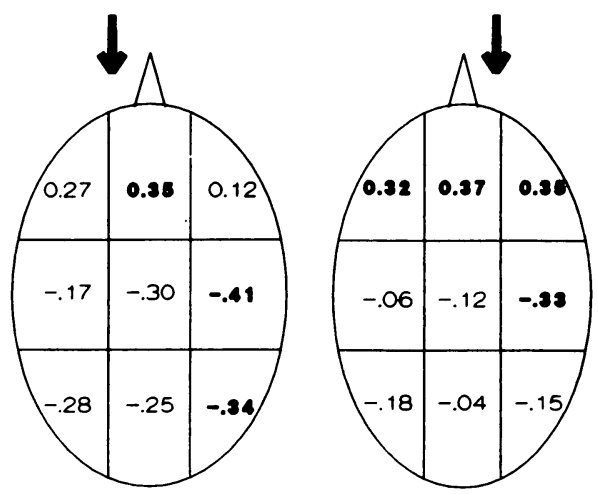

Figure 3. Correlations of peak P300 amplitude and CCCRC thresholds for each nostril at the various electrode locations. Values significant at the .05 level and beyond are in boldface.

CCCRC provides thresholds for each nostril, correlations for left and right nostril sensitivity and electrode position were obtained (see Figure 3). Positive correlations indicate associations of increased peak amplitude (positive) with increased sensitivity. Thus, subjects with the best odor sensitivity tended to have the highest positive peak amplitudes over midline frontal locations (Fz) and lowest amplitudes (or greatest negative amplitudes) over right temporal areas (T4), no matter which nostril was stimulated.

Peak and latency data for the ERP component occurring $0.32-0.52 \mathrm{sec}$ poststimulus were further examined in an analysis of variance (ANOVA) (BMDP 2V). The ANOVA revealed a dose-related main effect for odor intensity $[F(3,42)=4.52$, Greenhouse-Geisser $(\mathrm{G}-\mathrm{G}) p=.021]$, with the largest peak amplitudes occurring in response to the $4 \%$ condition. Interactions between intensity and electrode placement were also found such that amplitude was greatest along midline placements $[F(6,84)=4.38$, G-G $p$ $=.003]$ and greatest over posterior electrodes $[F(6,84)=$ $3.75, \mathrm{G}-\mathrm{G} p=.014]$. No odor-related effects were noted in the analyses of the latency measures. Grand mean waveforms for the midline electrode positions are presented in Figure 4 (4\% condition), and waveforms for each of the odor conditions at $\mathrm{Pz}$ are displayed in Figure 5.

As can be seen in Figures 4 and 5, much of the activity over the more posterior electrode positions was highly irregular and "noisy." Since subjects were tested with their eyes closed, high-amplitude alpha activity was present in the records of most of them, which contributed to the irregularity of the waveforms. Although this type of activity is undesirable, it was unavoidable in the present experiment because pilot studies had indicated that systematic eyeblinks often occur in this paradigm. Perhaps future adjustments to this paradigm can reduce such contamination.

The polarity, latency, and scalp distribution of the ERP component identified in this experiment suggests its similarity to the P300 component commonly reported in stimulus detection paradigms (Pritchard, 1981). Buttonpresses indicating detection of the odor tended to follow the peak of this ERP component by approximately $20 \mathrm{msec}$, as in 


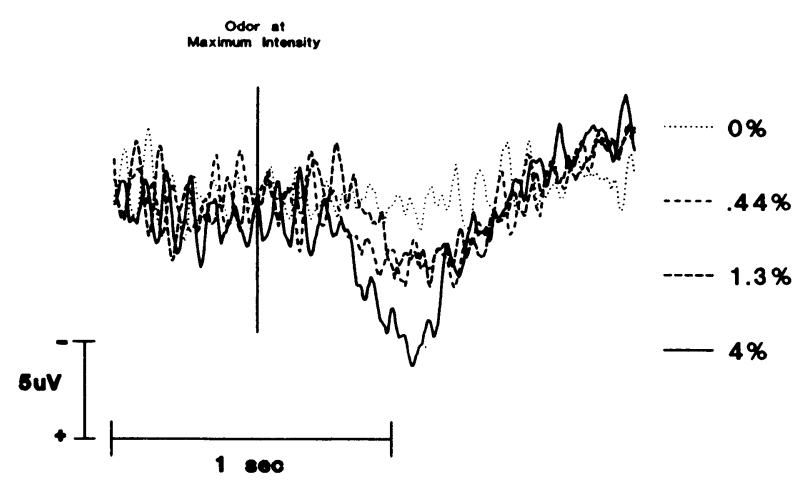

Figure 4. Grand mean ERPs at Pz for each of the four odor conditions.

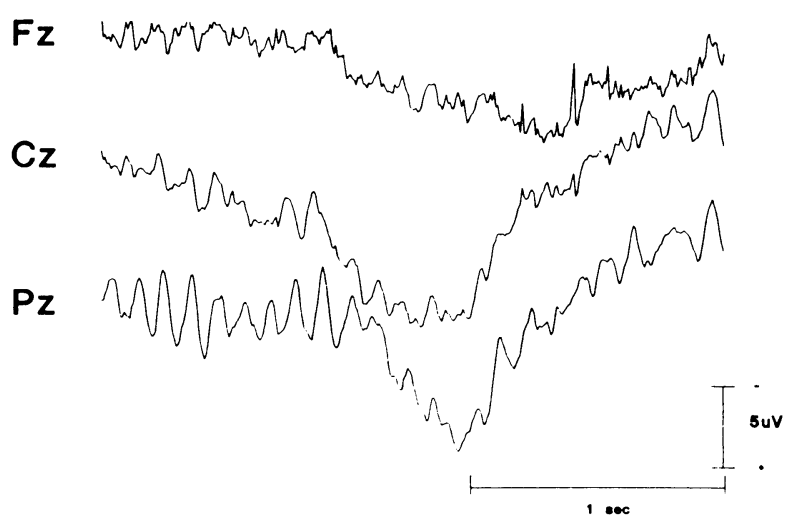

Figure 5. Grand mean ERPs for midline electrode placements for the $4 \%$ butanol condition.

other stimulus detection studies requiring overt responses. The presence of this consistent odor-related peak suggests that the paradigm used in its evocation is valid and that other components related to the perception and detection of odors should also be present. Surprisingly, no other identifiable peaks emerged in the PCA. Of special interest is the absence of an early negative component such as N100. Kobal and Hummel (1988) indicated an N100/P200 component of olfactory ERPs (maximal at $\mathrm{Cz}$ ) but found no P300 component. The disparity may be due to both paradigmatic and data compression differences in the studies. Because we employed a detection procedure with a no-odor condition, the occurrence of the P300 was optimized. This was not the case in Kobal and Hummel's (1988) experiment, in which subjects were not required to respond to the odor stimuli. Another difference in the two studies concerns the methods of averaging. In the present study, we employed linear averages with no latency adjustment, since previous findings obtained with latency adjustment procedures have proved unreliable (Lorig et al., 1988). Kobal et al. (1987) shifted the latency of the waveforms around an early negative peak, thus enhancing any early negativity.

Even though the first report of odor ERP occurred more than two decades ago, this form of ERP is still relatively unstudied. The technique holds promise for furthering clinical studies of individuals with olfactory dysfunction as well as identification of those with malingering anosmia. Further research to better define the respiration-based olfactory ERP is necessary. Latency adjustment procedures based on the amplitude or duration of respiration may help sharpen the averages obtained. In addition, different stimulation techniques such as the use of short nasal canuli may reduce variation in the waveform.

\section{REFERENCES}

Allison, T., \& GoFf, W. R. (1967). Human cerebral evoked responses to odorous stimuli. Electroencephalography \& Clinical Neurophysiology, 23, 558-560.

CaIN, W. S. (1989). Testing olfaction in a clinical setting. Ear, Nose \& Throat Journal, 68.

CaIN, W. S., \& RABIN, M. D. (1989). Comparability of two tests of olfactory functioning. Chemical Senses, 14, 479-485.

Doty, R. L., Deems, D. A., \& Stellar, S. (1988). Olfactory dysfunction in Parkinsonism: A general deficit unrelated to neurologic signs, disease stage, or duration. Neurology, 38, 1237-1244.

Elbert, T., Lutzenberger, W., Rockstroh, B., \& Birbaumer, N. (1985). Removal of occular artifacts from the EEG-A biophysical approach. Electroencephalography \& Clinical Neurophysiology, 60, 455-463.

Freeman, W. J., \& SChneider, W. (1982). Changes in the spatial patterns of rabbit olfactory EEG with conditioning to odors. Psychophysiology, 19, 44-56.

HJORTH, B. (1980). Source derivation simplifies topographical EEG interpretation. American Journal of EEG Technology, 20, 121-132.

Jones, B. P., Moskowitz, H. R, \& Butters, N. (1975). Olfactory quality discrimination in alcoholic Korsakoff patients. Neuropsychologia, 13, 173-179.

Kobal, G., \& Hummel, C. (1988). Cerebral chemosensory evoked potentials elicited by chemical stimulation of the human olfactory respiratory nasal mucosa. Electroencephalography \& Clinical Neurophysiology, 71, 241-250.

Kobal, G., Hummel, T., \& van Toller, C. (1987). Olfactory and chemo-somatosensory evoked potentials from stimuli presented to the left and right nostrils. Chemical Senses, 12, 183.

Koss, E. (1986). Olfactory dysfunction in Alzheimer's disease. Developmental Neuropsychology, 2, 89-99.

Lorig, T., Herman, K., \& Schwartz, G. (1988). Odor event-related potentials: Techniques and correlations with self-report. Psychophysiology, 25, 465-466.

Plattig, K.-H., \& Kobal, G. (1979). Spatial and temporal distribution of olfactory evoked potentials and techniques involved in their measure. In D. Lehmann \& E. Callaway (Eds.), Human evoked potentials (pp. 285-301). New York: Academic Press.

Pritchard, W. S. (1981). Psychophysiology of P300. Psychological Bulletin, 89, 506-540.

(Manuscript received October 9, 1992.) 